\title{
Expedited Management of Deep Vein Thrombosis and Acute Pulmonary Embolism
}

\author{
Stacy A. Johnson · Peter M. Yarbrough
}

Published online: 6 April 2013

(c) Springer Science+Business Media New York 2013

\begin{abstract}
Deep vein thrombosis and pulmonary embolism are common diseases with significant morbidity and mortality. Clinical probability assessment reliably identifies low-risk patients, and when paired with a negative D-dimer test, safely excludes venous thromboembolism. Compression ultrasonography confirms the diagnosis of deep vein thrombosis, whereas computed tomography pulmonary angiography is typically used to confirm pulmonary embolism. Risk assessment of acute pulmonary embolism stratifies low-risk patients for outpatient treatment, and high-risk patients for consideration of thrombolytic therapy. The recent approval of rivaroxaban, a novel oral anticoagulant, can simplify and expedite treatment in select patients. Ongoing clinical trials may uncover methods to prevent postthrombotic syndrome and chronic thromboembolic pulmonary hypertension. In this report, we review current diagnostic and management methods for deep vein thrombosis and pulmonary embolism.
\end{abstract}

Keywords Deep vein thrombosis · Pulmonary embolism . Diagnosis · Management $\cdot$ Risk stratification . Thrombolytic therapy · Antithrombotic therapy · Novel oral anticoagulant

S. A. Johnson $(\varangle)$ · P. M. Yarbrough

Department of Internal Medicine, University of Utah School of Medicine, 30 N 1900 E, Room 4B120, Salt Lake, UT 84132, USA

e-mail: stacy.a.johnson@hsc.utah.edu

P. M. Yarbrough

e-mail: peter.yarbrough@hsc.utah.edu

\section{Introduction}

Venous thromboembolism (VTE), comprising deep vein thrombosis (DVT) and pulmonary embolism (PE), is the third leading cause of cardiovascular mortality in the USA, responsible for nearly 550,000 hospitalizations annually, with DVT accounting for $60 \%$ of these events [1]. The 30-day mortality rate for PE is $8.2 \%$ with treatment and $20 \%$ with recurrent events [2]. Improved diagnostic techniques recently demonstrated one third of patients with DVT also have occult PE [3, 4]. In addition to disease burden, VTE also carries a significant financial burden for patients and the health care system. In patients with VTE, the 1 -year cost is estimated to be $\$ 33,531$, nearly twice that for matching controls [1].

The cornerstone of VTE treatment remains anticoagulation, which carries a considerable 3 -month bleeding risk of $2.1-12.8 \%[2,5 \bullet \cdot, 6]$. The difficulty lies in creating effective diagnostic and treatment strategies that detect VTE without placing patients at undue risk. This review outlines current diagnostic and management strategies for DVT and PE, focusing on established practices and the ever-changing landscape of this disease.

\section{Diagnosis}

Deep Vein Thrombosis

Limb swelling, pain, warmth, and erythema can all prompt DVT evaluation. Unfortunately, clinical assessment alone cannot accurately identify DVT [7]. Of all patients evaluated for DVT, only about $20 \%$ have the disease [5••]. Therefore, clinicians rely on multiple diagnostic methods, 
including clinical scoring systems, laboratory assays, and noninvasive imaging.

\section{Current Diagnostic Algorithm}

The University of Utah's evidence-based approach to DVT diagnosis is depicted in Fig. 1. This algorithm was derived from high-level studies, including risk prediction models, laboratory analysis, and different imaging modalities [contrast venography, two-point compression ultrasonography, whole-leg ultrasonography, computed tomography venography (CTV), and magnetic resonance venography (MRV)]. The components of this algorithm are based on influential reviews and consensus statements, including the current American College of Chest Physicians guidelines $[5 \bullet, 8,9,10,11]$. This algorithm combines pretest probability assessment with validated diagnostic methods to accurately diagnose or exclude DVT. A discussion of how this algorithm has evolved into our current approach and evolving issues including distal lower extremity DVT, upper extremity DVT (UEDVT), and bedside ultrasonography follows.

\section{Clinical Scoring Systems}

Although other scoring systems have been developed, the Wells score remains the most commonly used and widely validated method [12]. The simplified Wells score is illustrated in the text box in Fig. 1 [13]. A limitation of this scoring system is its reliance on the subjectivity of determining whether an alternative diagnosis is more or less likely than DVT. The initial diagnostic step is determination of DVT likelihood (Fig. 1). A score of 2 or greater indicates that DVT is likely and requires imaging, whereas a score less than 2 indicates DVT is unlikely, and one should test for D-dimer as the next step [13].

\section{D-Dimer}

D-dimer is a fibrin degradation product and its level is typically elevated in the presence of VTE [14]. However, the level of D-dimer is elevated in several clinical conditions, including infection, inflammation, cancer, surgery, trauma, burns, coronary artery disease, stroke, and pregnancy, limiting its positive predictive value [14]. Multiple D-dimer assays are available, with the highly sensitive D-dimer assays (ELISAs) demonstrating the best clinical utility [15]. For diagnosis of DVT, the D-dimer result must be combined with a clinical scoring system (Wells score) and ultrasonography, if indicated [13] (Fig. 1). Most importantly, a low pretest probability and a negative D-dimer test can safely exclude DVT without the need for imaging. A positive D-dimer test in patients with low pretest probability requires further evaluation before a diagnosis can be determined.

Imaging

Noninvasive compression ultrasonography (CUS) has essentially replaced contrast venography as the gold standard for diagnosis of DVT [16]. Two-point CUS, evaluating the common femoral and popliteal veins, has been extensively studied and widely adopted, despite being unable to detect distal DVT (calf vein) [17-19] (see the related article in this issue by Baird et al.) Up to $20 \%$ of distal DVTs propagate proximally in 1-2 weeks, necessitating serial CUS examinations [20]. Whole-leg CUS was later developed to detect proximal and distal DVT in a single study. The safety of this approach was demonstrated in a recent systematic review and meta-analysis for all patients, except those with the highest pretest probability [21]. A follow-up prospective study of high pretest probability patients with a negative whole-leg CUS result showed a similarly low VTE event rate of $0.6 \%$ at 3 months [22••]. On the basis of these results, whole-leg CUS is preferred over two-point CUS as it can safely exclude DVT in a single study. When whole-leg CUS is unavailable, an initial high pretest probability and negative two-point CUS result would require D-dimer testing and, if positive, another CUS examination in 1-2 weeks (Fig. 1). Other imaging modalities, including CTV [23], MRV [24, 25], and nuclear medicine scintigraphy [26, 27], may have benefits over CUS in select patients, but require additional study prior to being incorporated into routine DVT evaluation.

\section{Distal DVT}

Distal DVT is more commonly associated with transient risk factors and a lower risk of PE compared to proximal DVT. Some distal DVTs resolve without therapy, leading some clinicians to question their significance [28••]. However, in high-risk patients, 15-20\% of distal DVTs will extend proximally, necessitating treatment $[28 \bullet \bullet, 29]$. The increasing use of whole-leg CUS is likely attributing to more frequent diagnosis of distal DVT, and potentially unnecessary therapy.

\section{Upper Extremity DVT}

UEDVT refers to subclavian, axillary, or brachial vein thrombosis, and constitutes 4-10\% of all VTE. The frequent use of central venous catheters and cardiac devices may be responsible for the increasing prevalence of UEDVT $[11,30]$. Patients with UEDVT are more likely to be younger and thinner, and are at lower risk of PE (8 vs. $31 \%$ ) [31]. The literature on UEDVT is less extensive than that on lower extremity DVT, with insufficient data to guide management 


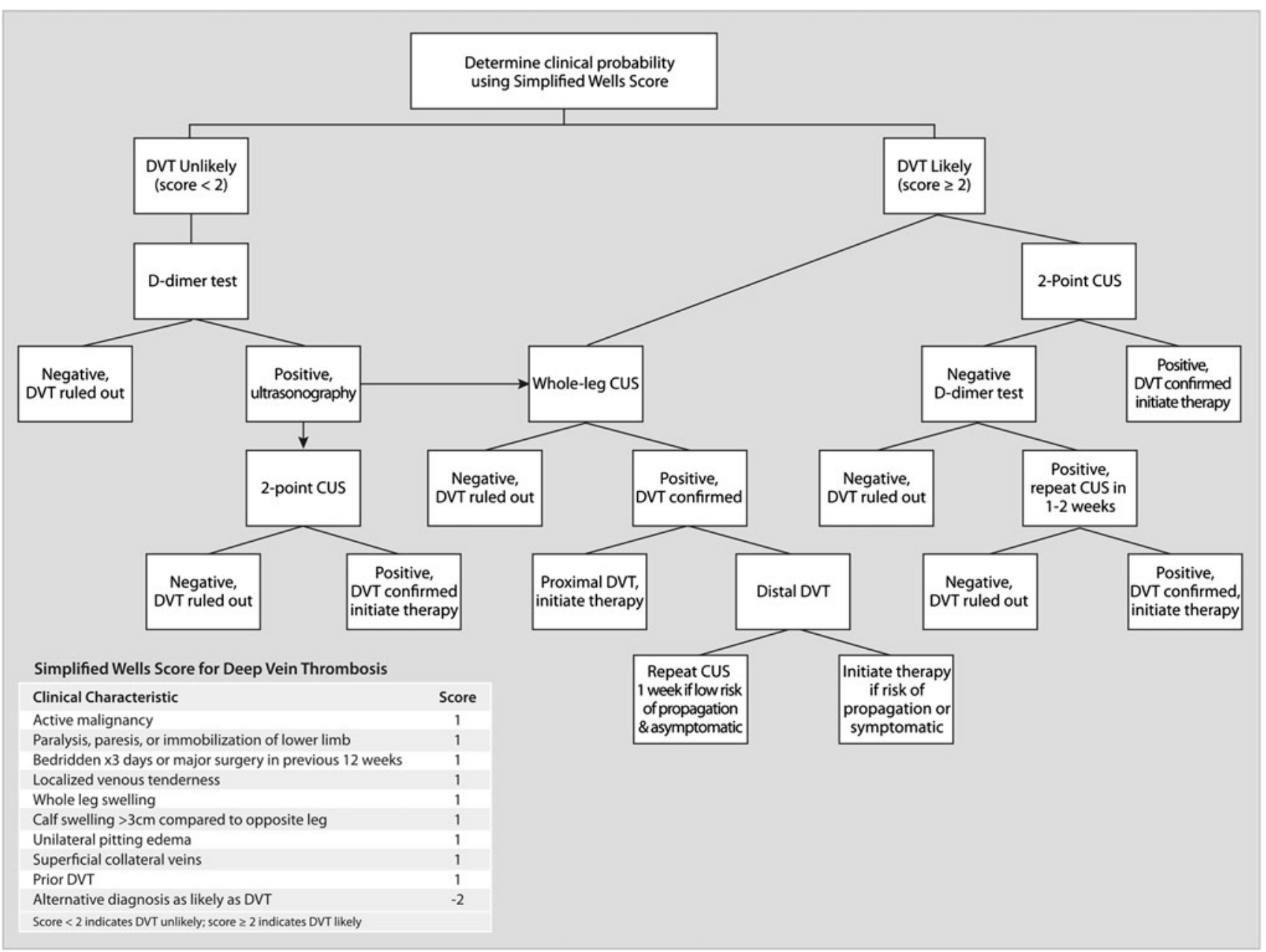

Fig. 1 Diagnostic algorithm for patients with suspected lower extremity deep vein thrombosis (DVT). CUS compression ultrasonography

[5••]. D-dimer has not been studied prospectively in UEDVT, and a negative ultrasonography result in a patient with high clinical suspicion is insufficient to exclude this diagnosis [30]. Therefore, high clinical suspicion with a negative CUS result warrants further evaluation. Contrast venography remains the gold standard [30]. CTV and MRV are less invasive than contrast venography, but there is insufficient evidence to justify widespread adoption of these imaging modalities [9].

\section{Bedside Ultrasonography}

Ultrasonography is becoming ubiquitous in emergency departments and more frequently available in hospitals for bedside use. There is considerable interest from clinicians to implement this technology for the prompt evaluation of suspected DVT. Emergency and critical care physicians can reliably diagnose DVT using bedside ultrasonography

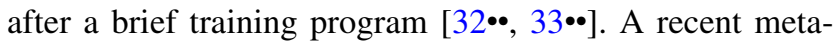
analysis of CUS performed in the emergency department for DVT showed $94.8 \%$ sensitivity and $96.2 \%$ specificity [34]. These studies demonstrate that a treating clinician can promptly diagnose and initiate DVT therapy, although further study is needed to validate this approach [34].

\section{Pulmonary Embolism}

Chest pain, shortness of breath, tachycardia, tachypnea, hypoxia, syncope, and hemoptysis can all be presenting signs and symptoms of PE. Much like DVT, the clinical presentation alone is insufficient to diagnose or exclude PE [35]. The diagnostic algorithm for PE is similar to that for DVT, as it incorporates clinical assessment, D-dimer testing, and imaging.

\section{Current Diagnostic Algorithm}

The University of Utah's evidence-based approach to PE diagnosis is outlined in Fig. 2. This approach combines a 
clinical scoring system with D-dimer testing and imaging, consistent with consensus guidelines [9, 36•]. The landmark study that reshaped PE diagnostics was the Christopher Study, which used a dichotomized Wells score with D-dimer testing and computed tomography pulmonary angiography (CTPA) to identify patients in whom PE was unlikely and anticoagulation could be safely withheld [37].

\section{Clinical Scoring Systems}

The Wells score for PE is the most used and best validated scoring system [38]. The current PE-specific Wells score uses a dichotomized score similar to the DVT scoring system, but relies on different clinical characteristics [37]. It has been criticized for the heavily weighted clinician assessment of "alternative diagnosis less likely than PE," which introduces a significant subjective component to the score. Regardless, the robust supporting evidence and ease of calculation make this scoring system the initial step for objectively assessing pretest probability for suspected PE.

The pulmonary embolism rule-out criteria (PERC) is an alternative method for pretest probability assessment in low-risk patients, as determined either by Wells criteria or by physician gestalt [39]. When all components of the rule are satisfied (low risk of PE, age less than 50 years, oxygen saturation $95 \%$ or greater, pulse rate below 100 beats per minute, no hemoptysis, no estrogen use, no hospitalization within 4 weeks for surgery/trauma, no prior VTE, and no unilateral leg swelling) the probability of VTE is less than $2 \%$ [38]. A recent meta-analysis of PERC estimated the pooled negative likelihood ratio to be 0.18 [95\% confidence interval (CI) 0.13-0.23] [40••].

\section{D-Dimer}

Mirroring DVT evaluation, D-dimer assessment is useful when the pretest probability is low and the result is negative, effectively ruling out PE [37]. The same limitations of D-dimer assays apply to D-dimer assessment in PE as well. Importantly, a negative D-dimer result cannot solely exclude the diagnosis of PE when the pretest probability is likely. Approximately $10 \%$ of patients with likely clinical probability and negative D-dimer result who were not anticoagulated suffered a VTE event [41]. Because the D-dimer test is not reliable enough to exclude PE in a patient with high likelihood, testing in such patients should begin with imaging (Fig. 2).

\section{Imaging}

CTPA has become the imaging modality of choice for PE diagnosis, essentially replacing the previous gold standard, pulmonary angiography $[23,37,42]$. When compared with ventilation-perfusion scanning, CTPA is noninferior and often provides alternative nonthrombotic diagnoses [42].

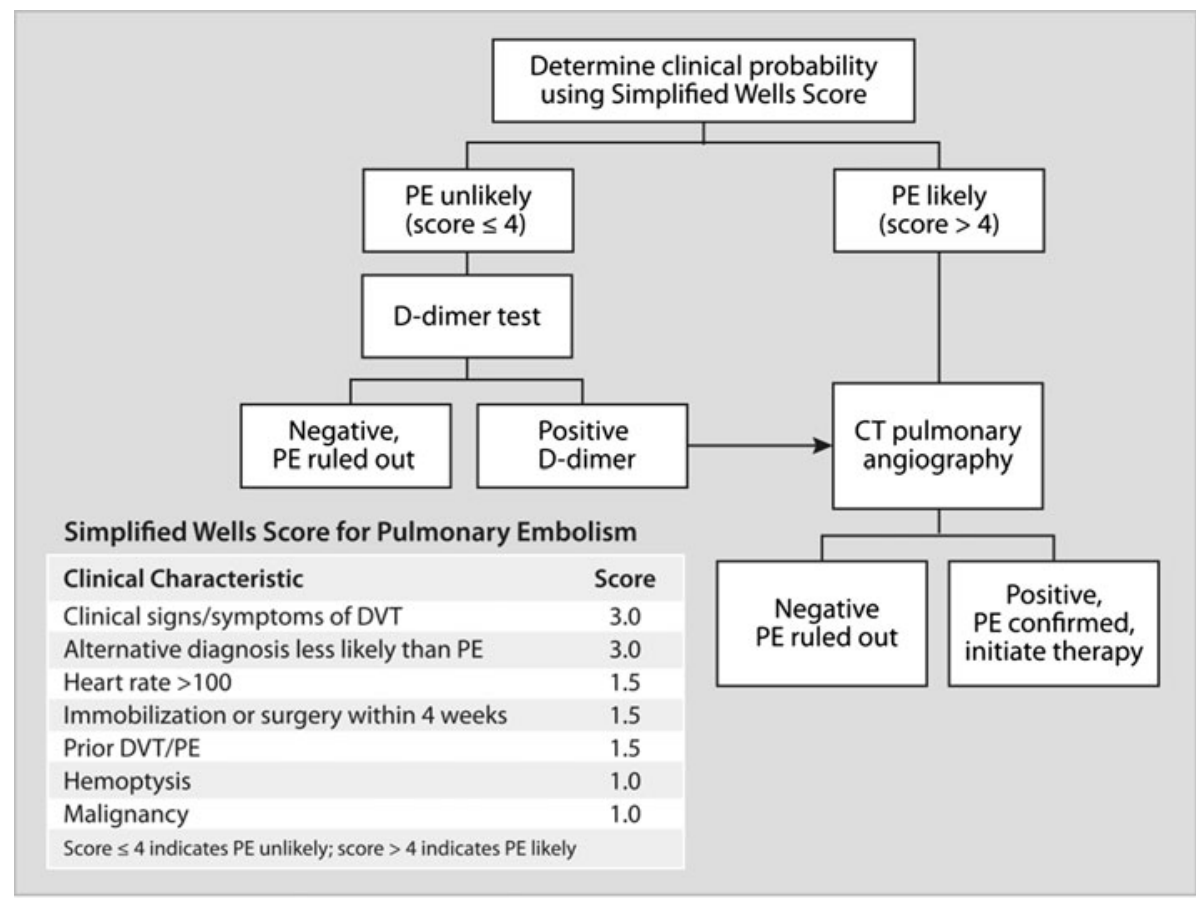

Fig. 2 Diagnostic algorithm for patients with suspected pulmonary embolism $(P E)$ 
Prospective studies demonstrate low VTE event rates $(0.27-1.3 \%)$ after a negative study $[37,43 \bullet \cdot$. The aforementioned studies offer considerable evidence to justify CTPA, combined with D-dimer testing and a clinical scoring system, as the standard of care for PE diagnosis. A negative CTPA result can safely exclude the diagnosis of PE (Fig. 2).

CTPA carries risks of radiation exposure, contrast allergies, and contrast-induced nephropathy. For these reasons, other imaging modalities remain relevant in PE diagnosis. Ventilation-perfusion scanning, originally compared with conventional pulmonary angiography in the PIOPED study [44], is reliable when combined with a clinical prediction model in patients with high or low probability scans. Unfortunately, scans are frequently of intermediate probability, requiring further diagnostic testing [42]. A patient with a normal chest X-ray may benefit from ventilation-perfusion scanning, given the decreased radiation exposure and fewer adverse effects compared with CTPA, if imaging is indicated [42].

Alternative imaging methods for PE include echocardiography [45], specialized nuclear medicine scans [46 ${ }^{\circ}$,

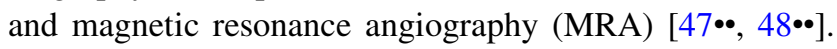
Echocardiography lacks adequate sensitivity $(80 \%)$ when compared with CTPA for routine diagnosis of PE [45]. Additionally, nuclear medicine scans lack sufficient data and MRA is plagued by a high rate of inconclusive studies and low sensitivity [48••], limiting their utility.

\section{Management}

Management of acute DVT and PE among hospitalized patients and those presenting to the emergency department continues to evolve. Advances in parenteral anticoagulation, risk stratification, and prevention strategies and the recent Food and Drug Administration approval of novel oral anticoagulants [49॰] continue to lower health care costs [50], broaden therapeutic options, and improve outcomes $[51 \cdot]$.

\section{PE Risk Stratification}

Acute PE is associated with a nearly fourfold increase in mortality compared with DVT [52]. Prompt risk stratification is essential during initial management, as prognosis depends on patient characteristics and clinical presentation [53]. Pulmonary emboli should be stratified into one of three risk categories-massive, submassive, or low-riskaccording to hemodynamic stability and evidence of right ventricular (RV) dysfunction [54••].
Definitions

Massive PE is defined as an acute PE with resultant arterial hypotension, either systolic blood pressure below $90 \mathrm{mmHg}$ or decrease in systolic pressure of $40 \mathrm{mmHg}$ or more, sustained over $15 \mathrm{~min}$ [55]. International registries support a link between arterial hypotension and adverse outcomes, with mortality rates ranging from 25 to $65 \%$ [56••, 57].

Submassive PE, defined as an acute PE with evidence of $\mathrm{RV}$ dysfunction and without arterial hypotension, carries a mortality rate of 3-15\% [58]. The presence of RV dysfunction appears to be associated with worse outcomes [59]. Several diagnostic techniques can identify RV dysfunction, including imaging, cardiac biomarkers, and electrocardiography [60]. Although the risk of RV failure is lower with submassive PE, identification of these patients is important to monitor them for early clinical deterioration and consideration of thrombolytic therapy [56••].

Low-risk PE, or nonmassive PE, is an acute PE without evidence of RV dysfunction or arterial hypotension [61]. These patients are at significantly lower risk of adverse outcomes (approximately $1 \%$ ) and generally have an excellent prognosis [54••]. Recent trials demonstrate select patients can be safely treated as outpatients [62•,63•].

\section{Clinical Scoring Systems}

The use of validated risk-prediction tools, such as the PE severity index [64], Geneva score [65], simplified PE severity index [66], or Hestia criteria [62.] can identify low-risk patients eligible for early hospital discharge or outpatient management [58, 63•]. Lankeit et al. [67] recently examined the combination of troponin $\mathrm{T}$ with the simplified PE severity index and demonstrated an increased ability to identify these low-risk patients. Future risk prediction models may rely on similar amalgamations of clinical assessment, biomarkers, and imaging.

Imaging

Noninvasive imaging modalities, including CTPA, echocardiography, and CUS, have been extensively studied, with conflicting results. CTPA is appealing as it can simultaneously diagnose PE and demonstrate RV dilation. Several studies suggest RV dilation on computed tomography is a predictor of adverse outcomes [68-70]. However, recent meta-analyses failed to substantiate these findings because of methodological diversity and limited prognostic value, questioning its utility for risk stratification $[59,71]$.

In addition to RV dilation, echocardiography can identify interventricular septal bowing, pulmonary arterial 
hypertension, and RV hypokinesis not identified by computed tomography [53, 72]. Normal RV function conveys a high negative predictive value $(98 \%)$ for all-cause mortality, PE-related mortality, and serious adverse events $[54 \cdot \bullet, 61,71]$. The availability of adequately trained echocardiography technicians and lack of standardized criteria have been cited as limitations [54••, 61]. However, bedside ultrasonography and echocardiography performed by emergency physicians can aid risk stratification [73, 74].

The utility of lower extremity DVT assessment at the time of PE diagnosis remains undetermined. Early studies failed to demonstrate a correlation between concurrent PE and DVT and mortality [72, 75]. Recently, a study demonstrated concurrent DVT is associated with increased mortality [15.2 vs. $6.4 \%$, hazard ratio (HR) 2.48] [76॰]. Further studies are needed to assess whether routine DVT screening at the time of PE diagnosis is cost-effective, or warrants additional therapy (thrombolytics or inferior vena caval filter).

\section{Biomarkers}

Several biomarkers have been evaluated for PE risk stratification [77-79]. Eleveation of the levels of troponin T and troponin I elevations can identify patients with submassive PE at increased risk of adverse outcomes (odds ratio 4.12) and mortality (odds ratio 5.90) [80]. Similarly, brain natriuretic peptide (BNP) and N-terminal pro-BNP can predict adverse events [59, 81]. A novel biomarker, growth differentiation factor 15 , outperformed troponin $\mathrm{T}$ and $\mathrm{N}$-terminal pro-BNP with respect to risk prediction of PE-related complications, deserving further study [82]. Cardiac biomarkers can supplement risk stratification in $\mathrm{PE}$, although sufficient predictive value to independently guide thrombolytic therapy is lacking [56••].

\section{Plasma Lactate}

Investigators from Florence, Italy, recently showed an increased plasma lactate level of $2 \mathrm{mmol} / \mathrm{L}$ or greater in patients with PE was associated with an increased risk of death (HR 11.67; $95 \%$ CI 3.32-41.03) [83]. This increased risk was independent of shock, RV dysfunction, or elevation of troponin I levels. Further study is needed to validate the role of elevated plasma lactate levels in PE risk stratification.

\section{Initial Antithrombotic Therapy}

The necessity of initial parenteral anticoagulation for DVT treatment has long been established [84]. In patients with normal renal function, unfractionated heparin (UFH) has largely been replaced by low molecular weight heparin (LMWH) and fondaparinux because of lower cost, lack of requisite laboratory monitoring, and favorable safety and efficacy profiles [85, 86*]. Meta-analysis of nearly 9,000 patients found LMWH superior to UFH with regard to allcause mortality, recurrent VTE, and major bleeding [87]. Fondaparinux demonstrated similar efficacy and safety profiles compared with LMWH, and superiority to UFH [56*0]. On the basis of these findings, current guidelines recommend LMWH and fondaparinux over UFH infusion $[56 \bullet \bullet$.

\section{Distal DVT}

As discussed already, the significance of distal DVT is unknown [28••, 29]. Current guidelines state if an isolated distal DVT is identified, then patient-specific risk factors and/or symptoms must be considered prior to initiation of anticoagulants [56••]. Severe symptoms (pain, swelling, erythema) or risk factors for thrombus propagation (positive D-dimer test, prior VTE, malignancy, immobilization, or extensive involvement of calf veins) warrant therapy. Patients without severe symptoms or risk factors for propagation should undergo serial CUS for 2 weeks to reassess them for propagation [5*0]. If thrombus extension is noted, antithrombotic therapy is recommended [56••].

\section{Proximal DVT}

In contrast to distal DVT, all proximal DVTs require treatment. Unless contraindicated, patients with proximal DVT should initiate parenteral anticoagulation for a minimum of 5 days, with concurrent vitamin $\mathrm{K}$ antagonist (VKA) therapy [56•*]. When contraindications to antithrombotic therapy exist, the patient should receive a removable inferior vena caval filter [56••]. If the contraindications are transient, then initiating anticoagulation and inferior vena caval filter removal are warranted on resolution of the contraindications $\left[56^{\bullet \bullet}\right]$.

\section{Upper Extremity DVT}

Management of UEDVT has not been as rigorously investigated as management of lower extremity DVT and PE [30]. At the time of writing, no randomized controlled therapeutic trials have been published. Retrospective and observational studies have demonstrated good outcomes with antithrombotic therapy $[88,89]$. On the basis of available evidence and lower extremity DVT experience, a similar treatment strategy is recommended [30, 56••]. This strategy consists of parenteral anticoagulation for a minimum of 5 days, and concurrent VKA administration. LMWHs are the preferred parenteral agents, except in 
patients with renal insufficiency, in whom UFH is suggested [90]. Discontinuation of use of central venous catheters is not essential if there is an ongoing need for the catheter and it remains functional $[30,56 \bullet \bullet]$.

\section{Pulmonary Embolism}

Prompt risk stratification of acute PE is essential to guide initial management. Massive PE warrants systemic thrombolytic therapy, catheter-assisted thrombolysis in patients with contraindications to systemic thrombolytic therapy, or surgical thrombectomy. Submassive and lowrisk PE therapy begins with parenteral anticoagulation for a minimum of 5 days and concurrent VKA administration. Generally, LMWHs are superior to UFH infusion, allowing early hospital discharge or outpatient therapy in low-risk patients $\left[56^{\bullet \bullet}, 63 \bullet\right]$.

\section{Maintenance Antithrombotic Therapy}

Maintenance therapy for UEDVT, lower extremity DVT, and PE is nearly identical. After an initial course of parenteral anticoagulation, maintenance antithrombotic therapy for at least 3 months is essential to prevent thrombus extension and recurrence. The decision to continue longterm therapy (beyond the first 3 months) should be individualized, dependent on patient-specific VTE-risk and bleeding-risk factors. Oral VKAs remain fundamental to maintenance therapy, despite their multiple limitations, such as narrow therapeutic window, requisite laboratory monitoring, myriad drug-drug and dietary interactions, and variable metabolism $[56 \bullet \bullet, 84]$. The intricacies of oral VKA management are beyond the scope of this review. Fortunately, advances in antithrombotic therapy are occurring.

\section{Novel Oral Anticoagulants}

Until recently, warfarin was the only oral anticoagulant available for VTE treatment in the USA [49•]. Several novel oral anticoagulants have been developed with differing pharmacologic properties and indications, outlined in Table 1. Some proposed advantages over VKAs include lack of required laboratory monitoring because of predictable pharmacology, fewer drug and dietary interactions, and with one agent initial parenteral anticoagulation appears unnecessary [51•,91]. Despite these potential advantages, clinicians should be aware of the limitations. No evidence-based reversal methods are available [92•]. Several drug interactions exist with inducers and inhibitors of cytochrome P450 3A4 and P-glycopeptide transporter systems (Table 1). Given most novel oral anticoagulants rely on both hepatic metabolism and renal clearance, patient selection is important to avoid excess bleeding risk [92•].

Evidence from the EINSTEIN trials demonstrated rivaroxaban was noninferior to standard therapy for the treatment of DVT (2.1 vs. $3.0 \%$; HR 0.68) and PE (2.1 vs. $1.8 \%$; HR 1.12). Bleeding risk was significantly lower in the EINSTEIN PE trial (HR 0.49; $95 \%$ CI, 0.31-0.79), but not significantly different in the DVT trials $[51 \bullet, 91]$. On the basis of the findings of these trials, rivaroxaban gained Food and Drug Administration approval for VTE treatment in November, 2012 [49॰]. Although the novel oral anticoagulants may offer prescribers attractive alternatives to standard therapy, more clinical experience is needed to justify widespread adoption.

\section{Thrombolytic Therapy}

Despite significant advances in the diagnosis and treatment of VTE, benefits of thrombolytic therapy remain in question. Thrombolytics have been assessed in multiple small randomized trials, meta-analyses, and registries with mixed results. Jaff et al. [54••] have provided a detailed summary of the available data.

\section{Massive PE}

Evidence-based guidelines advocate that patients with massive PE, without contraindications, should receive systemic thrombolytics [56••]. In patients with contraindications, or in whom systemic administration failed to improve hemodynamics, catheter-assisted thrombolysis is an alternative. This includes catheter-directed delivery of thrombolytics, mechanical thrombolysis, or a combination of these methods. An experienced operator should perform catheterassisted interventions. If an experience operator is locally unavailable, then urgent transfer to a capable institution is warranted [54••]. Although the evidence supporting these recommendations remains weak [56••], Wan et al. [93] demonstrated a significant reduction in the rates of death and recurrent $\mathrm{PE}$ (odds ratio 0.45 ) when analysis was limited to massive PE. Surgical thrombectomy is another effective strategy for management of massive PE, especially in those patients who have contraindications to thrombolytics or as a rescue option for those patients who continue to deteriorate despite use of thrombolytics [54••].

\section{Submassive PE}

In patients presenting with submassive PE and early clinical deterioration despite antithrombotic therapy, guidelines weakly recommend thrombolytic therapy [56••]. 
Table 1 Comparison of pharmacological properties and indications for novel oral anticoagulants

\begin{tabular}{|c|c|c|c|c|}
\hline & Rivaroxaban & Apixaban & Edoxaban & Dabigatran \\
\hline Mechanism of action & Factor Xa inhibitor & Factor Xa inhibitor & Factor Xa inhibitor & Direct thrombin inhibitor \\
\hline Half-life $T 1 / 2(\mathrm{~h})$ & $7-12$ & 13 & $9-11$ & $12-17$ \\
\hline Renal elimination (\%) & 66 & 25 & 35 & 80 \\
\hline Bioavailability (\%) & 80 & 60 & 50 & 5 \\
\hline Drug interactions & $\begin{array}{l}\text { Potent inhibitors } \\
\text { and inducers of } \\
\text { CYP3A4 and P-gp }\end{array}$ & $\begin{array}{l}\text { Potent inhibitors } \\
\text { and inducers of } \\
\text { CYP3A4 and P-gp }\end{array}$ & $\begin{array}{l}\text { Potent inhibitors } \\
\text { and inducers of } \\
\text { CYP3A4 and P-gp }\end{array}$ & $\begin{array}{l}\text { Potent inhibitors and } \\
\text { inducers of } \mathrm{P}-\mathrm{gp}^{\mathrm{b}}\end{array}$ \\
\hline Dosing & Once daily $^{c}$ & Twice daily & Once daily & Twice daily \\
\hline Reversal agent & None & None & None & None \\
\hline $\begin{array}{l}\text { Routine laboratory } \\
\text { monitoring }\end{array}$ & None & None & None & None \\
\hline $\begin{array}{l}\text { FDA-approved } \\
\text { indication(s) }\end{array}$ & $\begin{array}{l}\text { VTE treatment, VTE } \\
\text { prophylaxis after } \\
\text { TKA/THA, stroke } \\
\text { prophylaxis in } \\
\text { nonvalvular atrial } \\
\text { fibrillation }\end{array}$ & Pending & Pending & $\begin{array}{l}\text { Stroke } \\
\text { prophylaxis in } \\
\text { nonvalvular } \\
\text { atrial } \\
\text { fibrillation }\end{array}$ \\
\hline
\end{tabular}

CYP3A4 cytochrome P450 3A4, P-gp P-glycopeptide, VTE venous thromboembolism, TKA total knee arthroplasty, THA total hip arthroplasty, FDA Food and Drug Administration

${ }^{a}$ Potent combined CYP3A4 and P-gp inhibitors include ketoconazole, ritonavir, clarithromycin, erythromycin, and fluconazole; combined CYP3A4 and P-gp inducers include carbamazepine, phenytoin, rifampin, and St. John's wort

b Potent P-gp inhibitors include verapamil, amiodarone, quinidine, and clarithromycin

${ }^{c}$ Initial dosing in VTE treatment is twice daily for 3 weeks, followed by once daily. VTE prophylaxis and stroke prophylaxis dosing is once daily

Registry data demonstrate minimal mortality benefit of thrombolytics in submassive PE (less than $1 \%$ ) [54••]. Adjunctive thrombolytic therapy may confer some benefit over anticoagulation alone; potentially decreasing the development of chronic thromboembolic pulmonary hypertension and resulting in improvement in RV systolic pressure, RV function, 6-min walk test result, and New York Heart Association classification [94•, 95, 96]. Additional studies are ongoing to further assess the benefits of thrombolytics in submassive PE [97, 98].

\section{Deep Vein Thrombosis}

Systemic thrombolytics demonstrate greater thrombus reduction and fewer postthrombotic syndrome symptoms compared with anticoagulation alone [99]. However, major bleeding events (14 vs. $4 \%$ ) outweigh the benefits of systemic administration [54••]. In patients with severe symptoms or limb-threatening DVT (i.e., phlegmasia cerulea dolens), catheter-assisted thrombolysis is appropriate. Prior evidence on catheter-assisted thrombolysis demonstrated reductions in residual thrombus and postthrombotic syndrome symptoms, although the rate of major bleeding remained high (up to $11 \%$ ) [100]. Recently
Enden et al. [101] used a low-dose fibrinolytic regimen, with similar efficacy and fewer major bleeding events $(2.0 \%)$. A prospective trial comparing pharmacomechanical thrombolysis with anticoagulation alone for the prevention of postthrombotic syndrome is ongoing [102].

\section{Conclusions}

DVT and PE are common diseases, and are associated with significant morbidity and mortality. Clinical probability assessment with a validated risk prediction tool and adherence to diagnostic algorithms can expedite accurate diagnosis and treatment. Combining clinical assessment with a highly sensitive D-dimer test in certain populations can safely exclude VTE, reducing the use of unnecessary imaging studies. Prompt risk stratification of PE is important to identify patients eligible for outpatient therapy, or those requiring thrombolytic therapy. Novel oral anticoagulants, in properly selected patients, offer alternatives to standard therapy. Ongoing trials involving thrombolytic therapy for submassive PE and catheter-assisted thrombolysis for DVT may demonstrate reductions in the number of thromboembolic complications. 
Acknowledgments We sincerely thank Dr. Michael Lanspa for his excellent review and feedback on the manuscript. In addition, we thank Ms. Kim Mahoney for her assistance with the creation and design of the figures.

Disclosure S. Johnson: site principal investigator for Hokusai VTE Trial, Daiichi Sankyo Pharmaceuticals; P. Yarbrough: none.

\section{References}

Papers of particular interest, published recently, have been highlighted as:

- Of importance

•- Of major importance

1. Centers for Disease Control and Prevention. Venous thromboembolism in adult hospitalizations-United States, 2007-2009. MMWR Morb Mortal Wkly Rep. 2012;61(22):401-4.

2. Nijkeuter M, Sohne M, Tick LW, et al. The natural course of hemodynamically stable pulmonary embolism: clinical outcome and risk factors in a large prospective cohort study. Chest. 2007;131(2):517-23.

3. Stein PD, Matta F, Musani MH, Diaczok B. Silent pulmonary embolism in patients with deep venous thrombosis: a systematic review. Am J Med. 2010;123(5):426-31.

4. Tzoran I, Saharov G, Brenner B, et al. Silent pulmonary embolism in patients with proximal deep vein thrombosis in the lower limbs. J Thromb Haemost. 2012;10(4):564-71.

5. • Bates SM, Jaeschke R, Stevens SM, et al. Diagnosis of DVT: antithrombotic therapy and prevention of thrombosis. 9th ed. American College of Chest Physicians evidence-based clinical practice guidelines. Chest. 2012;141(2 Suppl):e351S-418S. Excellent reference and review of the diagnosis of DVT.

6. Hull RD, Raskob GE, Rosenbloom D, et al. Heparin for 5 days as compared with 10 days in the initial treatment of proximal venous thrombosis. N Engl J Med. 1990;322(18):1260-4.

7. Goodacre S, Sutton AJ, Sampson FC. Meta-analysis: the value of clinical assessment in the diagnosis of deep venous thrombosis. Ann Intern Med. 2005;143(2):129-39.

8. Goodacre S. In the clinic. Deep venous thrombosis. Ann Intern Med. 2008;149(5):ITC3-1.

9. Hogg K, Wells PS, Gandara E. The diagnosis of venous thromboembolism. Semin Thromb Hemost. 2012;38(7):691701.

10. Scarvelis D, Wells PS. Diagnosis and treatment of deep-vein thrombosis. CMAJ. 2006;175(9):1087-92.

11. Tan M, van Rooden CJ, Westerbeek RE, Huisman MV. Diagnostic management of clinically suspected acute deep vein thrombosis. Br J Haematol. 2009;146(4):347-60.

12. Tamariz LJ, Eng J, Segal JB, et al. Usefulness of clinical prediction rules for the diagnosis of venous thromboembolism: a systematic review. Am J Med. 2004;117(9):676-84.

13. Wells PS, Anderson DR, Rodger M, et al. Evaluation of D-dimer in the diagnosis of suspected deep-vein thrombosis. N Engl $\mathrm{J}$ Med. 2003;349(13):1227-35.

14. Righini M, Perrier A, De Moerloose P, Bounameaux H. D-dimer for venous thromboembolism diagnosis: 20 years later. J Thromb Haemost. 2008;6(7):1059-71.

15. Stein PD, Hull RD, Patel KC, et al. D-dimer for the exclusion of acute venous thrombosis and pulmonary embolism: a systematic review. Ann Intern Med. 2004;140(8):589-602.
16. Dauzat M, Laroche JP, Deklunder G, et al. Diagnosis of acute lower limb deep venous thrombosis with ultrasound: trends and controversies. J Clin Ultrasound. 1997;25(7):343-58.

17. Cogo A, Lensing AW, Koopman MM, et al. Compression ultrasonography for diagnostic management of patients with clinically suspected deep vein thrombosis: prospective cohort study. BMJ. 1998;316(7124):17-20.

18. Lensing AW, Prandoni P, Brandjes D, et al. Detection of deepvein thrombosis by real-time B-mode ultrasonography. N Engl J Med. 1989;320(6):342-5.

19. Kearon C, Ginsberg JS, Douketis J, et al. A randomized trial of diagnostic strategies after normal proximal vein ultrasonography for suspected deep venous thrombosis: D-dimer testing compared with repeated ultrasonography. Ann Intern Med. 2005; 142(7):490-6.

20. Hirsh J. How we diagnose and treat deep vein thrombosis. Blood. 2002;99(9):3102-10.

21. Johnson SA, Stevens SM, Woller SC, et al. Risk of deep vein thrombosis following a single negative whole-leg compression ultrasound: a systematic review and meta-analysis. JAMA. 2010;303(5):438-45.

22. • Stevens SM, Woller SC, Graves KK, et al. Withholding anticoagulation following a single negative whole-leg ultrasound in patients at high pretest probability for deep vein thrombosis. Clin Appl Thromb Hemost. 2013;19(1):79-85. A diagnostic management study looking at high pretest probability patients and negative whole-leg ultrasound. 3 month VTE rate was $0.60 \%$ making this a safe management strategy for these patients.

23. Stein PD, Fowler SE, Goodman LR, et al. Multidetector computed tomography for acute pulmonary embolism. N Engl J Med. 2006;354(22):2317-27.

24. Fraser DG, Moody AR, Morgan PS, et al. Diagnosis of lowerlimb deep venous thrombosis: a prospective blinded study of magnetic resonance direct thrombus imaging. Ann Intern Med. 2002;136(2):89-98.

25. Carpenter JP, Holland GA, Baum RA, et al. Magnetic resonance venography for the detection of deep venous thrombosis: comparison with contrast venography and duplex doppler ultrasonography. J Vasc Surg. 1993;18(5):734-41.

26. Douketis JD, Ginsberg JS, Haley S, et al. Accuracy and safety of ${ }^{99 \mathrm{~m}}$ Tc-labeled anti-D-dimer (DI-80B3) Fab' fragments (ThromboView $\left.{ }^{\circledR}\right)$ in the diagnosis of deep vein thrombosis: a phase II study. Thromb Res. 2012;130(3):381-9.

27. Rondina MT, Lam UT, Pendleton RC, et al. 18F-FDG PET in the evaluation of acuity of deep vein thrombosis. Clin Nucl Med. 2012;37(12):1139-45.

28. • Palareti G, Cosmi B, Lessiani G, et al. Evolution of untreated calf deep-vein thrombosis in high risk symptomatic outpatients: the blind, prospective CALTHRO study. Thromb Haemost. 2010;104(5):1063-70. Excellent recent review of this very pertinent and evolving clinical condition.

29. Palareti G, Schellong S. Isolated distal deep vein thrombosis: what we know and what we are doing. J Thromb Haemost. 2012;10(1):11-9.

30. Grant JD, Stevens SM, Woller SC, et al. Diagnosis and management of upper extremity deep-vein thrombosis in adults. Thromb Haemost. 2012;108(6):1097-108.

31. Lechner D, Wiener C, Weltermann A, et al. Comparison between idiopathic deep vein thrombosis of the upper and lower extremity regarding risk factors and recurrence. J Thromb Haemost. 2008;6(8):1269-74.

32. • Crisp JG, Lovato LM, Jang TB. Compression ultrasonography of the lower extremity with portable vascular ultrasonography can accurately detect deep venous thrombosis in the emergency department. Ann Emerg Med. 2010;56(6):601-10. A prospective 
accuracy study of ED performed ultrasound after 10 minutes of education. $100 \%$ sensitive and $99 \%$ specific.

33. •- Kory PD, Pellecchia CM, Shiloh AL, et al. Accuracy of ultrasonography performed by critical care physicians for the diagnosis of DVT. Chest. 2011;139(3):538-42. Accuracy study of critical care physician ultrasound in the diagnosis of DVT. $86 \%$ sensitivity and $96 \%$ specificity. Furthering the body of literature supporting bedside ultrasound in the rapid diagnosis of DVT.

34. Pomero F, Dentali F, Borretta V, et al. Accuracy of emergency physician-performed ultrasonography in the diagnosis of deepvein thrombosis. A systematic review and meta-analysis. Thromb Haemost. 2012;109(1):137-45.

35. Stein PD, Beemath A, Matta F, et al. Clinical characteristics of patients with acute pulmonary embolism: data from PIOPED II. Am J Med. 2007;120(10):871-9.

36. - Agnelli G, Becattini C. Acute pulmonary embolism. N Engl J Med. 2010;363(3):266-74. A good recent review of PE.

37. van Belle A, Buller HR, Huisman MV, et al. Effectiveness of managing suspected pulmonary embolism using an algorithm combining clinical probability, D-dimer testing, and computed tomography. JAMA. 2006;295(2):172-9.

38. Kline JA, Mitchell AM, Kabrhel C, et al. Clinical criteria to prevent unnecessary diagnostic testing in emergency department patients with suspected pulmonary embolism. J Thromb Haemost. 2008;6(5):772-80.

39. Singh B, Parsaik AK, Agarwal D, et al. Diagnostic accuracy pulmonary embolism rule-out criteria: a systematic review and meta-analysis. Ann Emerg Med. 2012;59:517-20.

40. $\bullet$ Penaloza A, Melot C, Motte S. Comparison of the wells score with the simplified revised geneva score for assessing pretest probability of pulmonary embolism. Thromb Res. 2011;127(2): 81-4. A comparison of the Wells score and revised Geneva score for patients with PE. Wells score found to be more accurate.

41. Gibson NS, Sohne M, Gerdes VE, et al. The importance of clinical probability assessment in interpreting a normal d-dimer in patients with suspected pulmonary embolism. Chest. 2008;134(4):789-93.

42. Anderson DR, Kahn SR, Rodger MA, et al. Computed tomographic pulmonary angiography versus ventilation-perfusion lung scanning in patients with suspected pulmonary embolism: a randomized controlled trial. JAMA. 2007;298(23):2743-53.

43. •- Pesavento R, de Conti G, Minotto I, et al. The value of 64-detector row computed tomography for the exclusion of pulmonary embolism. Thromb Haemost. 2011;105(5):901-7. A prospective management study using the Christopher Study algorithm and 64-detector row CT scan. Found to have a reduced rate of VTE at 3 months $(0.27 \%)$ than the Christopher Study which used less advanced CT techonology.

44. Value of the ventilation/perfusion scan in acute pulmonary embolism. Results of the prospective investigation of pulmonary embolism diagnosis (PIOPED). The PIOPED Investigators JAMA. 1990;263(20):2753-9.

45. Goldhaber SZ. Echocardiography in the management of pulmonary embolism. Ann Intern Med. 2002;136(9):691-700.

46. - Morris TA, Gerometta M, Yusen RD, et al. Detection of pulmonary emboli with 99mTc-labeled anti-D-dimer (DI-80B3) Fab' fragments (ThromboView). Am J Respir Crit Care Med. 2011;184(6):708-14. An accuracy study looking at a novel technique to diagnose PE Sensitivity was $76.2 \%$ and specificity was $90.5 \%$, but kappa was only 0.62 for this technique between providers.

47. •- Stein PD, Chenevert TL, Fowler SE, et al. Gadoliniumenhanced magnetic resonance angiography for pulmonary embolism: a multicenter prospective study (PIOPED III). Ann
Intern Med. 2010;152(7):434-43, W142-3. This made sensitivity and specificity low. However, in those patients with adequate images, sensitivity and specificity were $92 \%$ and $96 \%$ respectively.

48. $\bullet$ Revel MP, Sanchez O, Couchon S, et al. Diagnostic accuracy of magnetic resonance imaging for an acute pulmonary embolism: results of the 'IRM-EP' study. J Thromb Haemost. 2012;10(5):743-50. Accuracy study looking at MRA compared to CTA. Negative predictive value remains too low to be used to exclude PE.

49. - FDA expands use of Xarelto to treat, reduce recurrence of blood clots. http://www.fda.gov/NewsEvents/Newsroom/Press Announcements/ucm326654.htm. Accessed 2 Nov 2012. Recent FDA announcement of its approval of rivaroxaban for the treatment of venous thromboembolism.

50. Gussoni G, Foglia E, Frasson S, et al. Real-world economic burden of venous thromboembolism and antithrombotic prophylaxis in medical inpatients. Thromb Res. 2012;131(1): $17-23$.

51. - Buller HR, Prins MH, Lensin AW, et al. Oral rivaroxaban for the treatment of symptomatic pulmonary embolism. N Engl J Med. 2012;366(14):1287-97. Einstein PE trial demonstrating the noninferiority of rivaroxaban compared to standard therapy, and superiority with respect to bleeding events.

52. Douketis JD, Kearon C, Bates S, et al. Risk of fatal pulmonary embolism in patients with treated venous thromboembolism. JAMA. 1998;279(6):458-62.

53. Grifoni S, Olivotto I, Cecchini P, et al. Short-term clinical outcome of patients with acute pulmonary embolism, normal blood pressure, and echocardiographic right ventricular dysfunction. Circulation. 2000;101(24):2817-22.

54. • Jaff MR, McMurtry MS, Archer SL, et al. Management of massive and submassive pulmonary embolism, iliofemoral deep vein thrombosis, and chronic thromboembolic pulmonary hypertension: a scientific statement from the American Heart Association. Circulation. 2011;123(16):1788-830. Recent comprehensive AHA guidelines on the treatment of iliofemoral DVT, submassive PE, massive PE, and chronic thromboembolic pulmonary hypertension.

55. Kucher N, Goldhaber SZ. Management of massive pulmonary embolism. Circulation. 2005;112(2):e28-32.

56. •- Kearon C, Akl EA, Comerota AJ, et al. Antithrombotic therapy for VTE disease: antithrombotic therapy and prevention of thrombosis. 9th ed. American College of Chest Physicians evidence-based clinical practice guidelines. Chest. 2012;141(2 Suppl):e419S-94S. The most recent and most comprehensive published guidelines on VTE treatment.

57. Kucher N, Rossi E, De Rosa M, Goldhaber SZ. Massive pulmonary embolism. Circulation. 2006;113(4):577-82.

58. Aujesky D, Stone RA, Kim S, et al. Length of hospital stay and postdischarge mortality in patients with pulmonary embolism: a statewide perspective. Arch Intern Med. 2008;168(7):706-12.

59. Sanchez O, Trinquart L, Colombet I, et al. Prognostic value of right ventricular dysfunction in patients with haemodynamically stable pulmonary embolism: a systematic review. Eur Heart J. 2008;29(12):1569-77.

60. Piazza G, Goldhaber SZ. Management of submassive pulmonary embolism. Circulation. 2010;122(11):1124-9.

61. Penaloza A, Roy PM, Kline J. Risk stratification and treatment strategy of pulmonary embolism. Curr Opin Crit Care. 2012; 18(4):318-25.

62. - Zondag W, Mos IC, Creemers-Schild D, et al. Outpatient treatment in patients with acute pulmonary embolism: the Hestia Study. J Thromb Haemost. 2011;9(8):1500-7. This is a study of 297 patients with low-risk PE demonstrating in selected patients, outpatient therapy is safe and effective. 
63. - Howard L, Salooja N. Outpatient management of pulmonary embolism. Lancet. 2011;378(9785):5-6. Randomized trial comparing inpatient vs. outpatient treatment for patients with low-risk PE demonstrating outpatient treatment is as safe and effective as inpatient therapy in these selected patients.

64. Aujesky D, Obrosky DS, Stone RA, et al. Derivation and validation of a prognostic model for pulmonary embolism. Am J Respir Crit Care Med. 2005;172(8):1041-6.

65. Wicki J, Perrier A, Perneger TV, et al. Predicting adverse outcome in patients with acute pulmonary embolism: a risk score. Thromb Haemost. 2000;84(4):548-52.

66. Jimenez D, Aujesky D, Moores L, et al. Simplification of the pulmonary embolism severity index for prognostication in patients with acute symptomatic pulmonary embolism. Arch Intern Med. 2010;170(15):1383-9.

67. Lankeit M, Jimenez D, Kostrubiec M, et al. Predictive value of the high-sensitivity troponin $\mathrm{T}$ assay and the simplified pulmonary embolism severity index in hemodynamically stable patients with acute pulmonary embolism: a prospective validation study. Circulation. 2011;124(24):2716-24.

68. Schoepf UJ, Kucher N, Kipfmueller F, et al. Right ventricular enlargement on chest computed tomography: a predictor of early death in acute pulmonary embolism. Circulation. 2004;110(20): 3276-80.

69. Stein PD, Beemath A, Matta F, et al. Enlarged right ventricle without shock in acute pulmonary embolism: prognosis. Am $\mathbf{J}$ Med. 2008;121(1):34-42.

70. Nural MS, Elmali M, Findik S, et al. Computed tomographic pulmonary angiography in the assessment of severity of acute pulmonary embolism and right ventricular dysfunction. Acta Radiol. 2009;50(6):629-37.

71. Coutance G, Cauderlier E, Ehtisham J, et al. The prognostic value of markers of right ventricular dysfunction in pulmonary embolism: a meta-analysis. Crit Care. 2011;15(2):R103.

72. Goldhaber SZ, Visani L, De Rosa M. Acute pulmonary embolism: clinical outcomes in the International Cooperative Pulmonary Embolism Registry (ICOPER). Lancet. 1999;353(9162): 1386-9.

73. Borloz MP, Frohna WJ, Phillips CA, Antonis MS. Emergency department focused bedside echocardiography in massive pulmonary embolism. J Emerg Med. 2011;41(6):658-60.

74. Misiaszek RA, Budhram G. Diagnosis of pulmonary embolism using emergency department bedside echocardiogram. Acad Emerg Med. 2009;16(2):188-9.

75. Girard P, Sanchez O, Leroyer C, et al. Deep venous thrombosis in patients with acute pulmonary embolism: prevalence, risk factors, and clinical significance. Chest. 2005;128(3):1593-600.

76. - Jimenez D, Aujesky D, Diaz G, et al. Prognostic significance of deep vein thrombosis in patients presenting with acute symptomatic pulmonary embolism. Am J Respir Crit Care Med. 2010;181(9):983-91. New evidence that patients presenting with acute PE found to have simultaneous DVT are at increased risk for PE related death and all cause mortality in the ensuing 3 months.

77. Kline JA, Zeitouni R, Marchick MR, et al. Comparison of 8 biomarkers for prediction of right ventricular hypokinesis 6 months after submassive pulmonary embolism. Am Heart J. 2008;156(2):308-14.

78. Dellas C, Puls M, Lankeit M, et al. Elevated heart-type fatty acid-binding protein levels on admission predict an adverse outcome in normotensive patients with acute pulmonary embolism. J Am Coll Cardiol. 2010;55(19):2150-7.

79. Ghanima W, Abdelnoor M, Holmen LO, et al. D-dimer level is associated with the extent of pulmonary embolism. Thromb Res. 2007;120(2):281-8.
80. Becattini C, Vedovati MC, Agnelli G. Prognostic value of troponins in acute pulmonary embolism: a meta-analysis. Circulation. 2007;116(4):427-33.

81. Cavallazzi R, Nair A, Vasu T, Marik PE. Natriuretic peptides in acute pulmonary embolism: a systematic review. Intensive Care Med. 2008;34(12):2147-56.

82. Lankeit M, Kempf T, Dellas C, et al. Growth differentiation factor-15 for prognostic assessment of patients with acute pulmonary embolism. Am J Respir Crit Care Med. 2008; 177(9):1018-25.

83. Vanni S, Viviani G, Baioni M, et al. Prognostic value of plasma lactate levels among patients with acute pulmonary embolism: the thrombo-embolism lactate outcome study. Ann Emerg Med. 2013;61:330-8.

84. Brandjes DP, Heijboer H, Buller HR, et al. Acenocoumarol and heparin compared with acenocoumarol alone in the initial treatment of proximal-vein thrombosis. N Engl J Med. 1992; 327(21):1485-9.

85. Gould MK, Dembitzer AD, Sanders GD, Garber AM. Lowmolecular-weight heparins compared with unfractionated heparin for treatment of acute deep venous thrombosis. A costeffectiveness analysis. Ann Intern Med. 1999;130(10):78-99.

86. - Erkens PM, Prins MH. Fixed dose subcutaneous low molecular weight heparins versus adjusted dose unfractionated heparin for venous thromboembolism. Cochrane Database Syst Rev. 2010;8(9):CD001100. Largest meta-analysis to date including more than 9,000 patients demonstrating LMWH is superior to UFH with respect to recurrent VTE events, major bleeding, and mortality, making LMWHs the parenteral anticoagulants of choice for patients with acute VTE and normal renal function.

87. van Dongen CJ, van den Belt AG, Prins MH, Lensing AW. Fixed dose subcutaneous low molecular weight heparins versus adjusted dose unfractionated heparin for venous thromboembolism. Cochrane Database Syst Rev. 2004;4:CD001100.

88. Savage KJ, Wells PS, Schulz V, et al. Outpatient use of low molecular weight heparin (dalteparin) for the treatment of deep vein thrombosis of the upper extremity. Thromb Haemost. 1999;82(3):1008-10.

89. Hingorani A, Ascher E, Ward M, et al. Combined upper and lower extremity deep venous thrombosis. Cardiovasc Surg. 2001;9(5):472-7.

90. Kucher N. Clinical practice. Deep-vein thrombosis of the upper extremities. N Engl J Med. 2011;364(9):861-9.

91. Bauersachs R, Berkowitz SD, Brenner B, et al. Oral rivaroxaban for symptomatic venous thromboembolism. N Engl J Med. 2010;363(26):2499-510.

92. - Garcia D, Libby E, Crowther MA. The new oral anticoagulants. Blood. 2010;115(1):15-20. Comprehensive review of available evidence on novel oral anticoagulants.

93. Wan S, Quinlan DJ, Agnelli G, Eikelboom JW. Thrombolysis compared with heparin for the initial treatment of pulmonary embolism: a meta-analysis of the randomized controlled trials. Circulation. 2004;110(6):744-9.

94. - Fasullo S, Scalzo S, Maringhini G, et al. Six-month echocardiographic study in patients with submassive pulmonary embolism and right ventricle dysfunction: comparison of thrombolysis with heparin. Am J Med Sci. 2011;341(1):33-9. Study demonstrating early and sustained improvement in $R V$ function after six months on echocardiography in patients with submassive PE treated with thrombolytic therapy.

95. Kline JA, Steuerwald MT, Marchick MR, et al. Prospective evaluation of right ventricular function and functional status 6 months after acute submassive pulmonary embolism: frequency of persistent or subsequent elevation in estimated pulmonary artery pressure. Chest. 2009;136(5):1202-10. 
96. Sharma GV, Folland ED, McIntyre KM, Sasahara AA. Longterm benefit of thrombolytic therapy in patients with pulmonary embolism. Vasc Med. 2000;5(2):91-5.

97. PEITHO pulmonary embolism thrombolysis study. http:// clinicaltrials.gov/ct2/show/NCT00639743?term=tenecteplase \& rank=15. Accessed 2 Nov 2012.

98. Clot dissolving treatment for blood clots in the lungs. http:// clinicaltrials.gov/ct2/show/NCT00680628?term=tenecteplase \& rank=11. Accessed 2 Nov 2012.

99. Goldhaber SZ, Buring JE, Lipnick RJ, Hennekens CH. Pooled analyses of randomized trials of streptokinase and heparin in phlebographically documented acute deep venous thrombosis. Am J Med. 1984;76(3):393-7.
100. Mewissen MW, Seabrook GR, Meissner MH, et al. Catheterdirected thrombolysis for lower extremity deep venous thrombosis: report of a national multicenter registry. Radiology. 1999;211(1):39-49.

101. Enden T, Klow NE, Sandvik L, et al. Catheter-directed thrombolysis versus anticoagulant therapy alone in deep vein thrombosis: results of an open randomized, controlled trial reporting on short-term patency. J Thromb Haemost. 2009;7(8):1268-75.

102. ATTRACT trial. http://clinicaltrials.gov/ct2/show/study/NCT00 790335?term=ATTRACT\&rank=1. Accessed 12 Dec 2012. 